Gastroenteropancreatic neuroendocrine tumors (GEP-NETs) are a large and very diverse group of neoplasms. Clinical presentation of NETs depends on the site of the primary tumor and whether the tumor is functioning (i.e., secreting peptides or neuroamines that produce symptoms). The diagnosis of GEP-NET is further complicated by symptomatic differences that occur depending on the type of secreted peptide or neuroamine. Due to their heterogeneity and unique characteristics, early diagnosis of GEP-NETs is difficult, which increases the likelihood of metastatic disease and reduces the scope of therapeutic possibilities. Thus, a multidisciplinary approach for the treatment of GEPNETs is necessary. This review is the result of presentations that were delivered during an expert meeting on the treatment of GEP-NETs supported by Ipsen. We summarize the current knowledge on the epidemiology, incidence, diagnosis, and treatment of GEP-NETs. We examined the role of the somatostatin analog (SSA) lanreotide and the impact of the data from the recently published, randomized, double-blind, placebo-controlled CLARINET study (Controlled study of Lanreotide Antiproliferative Response In Neuroendocrine Tumors) on disease management. We also review the recent treatment options and recommendations for GEP-NETs.

Key words: gastroenteropancreatic neuroendocrine tumors, GEP-NETs, somatostatin analogue, lanreotide, CLARINET.

Contemp Oncol (Pozn) 2017; 21 (2): 115-122 DOI: https://doi.org/10.5114/wo.2017.68619

\section{Current treatment options for gastroenteropancreatic neuroendocrine tumors with a focus on the role of lanreotide}

\author{
Beata Kos-Kudła' ${ }^{1}$ Jarosław Ćwikła ${ }^{2}$, Marek Ruchała ${ }^{3}$, Alicja Hubalewska- \\ -Dydejczyk ${ }^{4}$, Barbara Jarzab ${ }^{5}$, Jolanta Krajewska ${ }^{5}$, Grzegorz Kamiński ${ }^{6}$
}

${ }^{1}$ Division of Endocrinology, Department of Pathophysiology and Endocrinology, Silesian Medical University, Katowice, Poland

${ }^{2}$ Department of Medical Science, University of Varmia \& Masuria, Olsztyn, Poland ${ }^{3}$ Department of Endocrinology, Metabolism and Internal Medicine, Poznan University of Medical Sciences, Poznan, Poland

${ }^{4}$ Department of Endocrinology, Jagiellonian University Medical College, Cracow, Poland ${ }^{5}$ Department of Nuclear Medicine and Endocrine Oncology, M. Sklodowska-Curie Memorial Cancer Center and Institute of Oncology, Gliwice, Poland ${ }^{6}$ Department of Endocrinology and Isotope Therapy, Military Institute of Medicine, Warsaw, Poland

\section{Epidemiology and prevalence of neuroendocrine tumors}

Gastroenteropancreatic neuroendocrine tumors (GEP-NETs) are a heterogeneous group of neoplasms arising from highly specialized cells of the diffuse endocrine system [1]. The characteristic feature of NETs is their ability to synthesize, store, and secrete peptides, hormones, and neuroamines. According to global statistics reported in 2010, the average prevalence of NETs is 5.25 cases per 100000 population per year, with the risk increasing after 50 years of age [2]. GEP tumors account for $70 \%$ of all NETs and about $2 \%$ of all gastrointestinal system tumors [1].

GEP-NET primary lesions are most commonly localized in the gastric mucosa, the small and large intestine, the rectum, or the pancreas [1]. According to the American Surveillance Epidemiology and End Results (SEER) database, the incidence per year is 0.95 per 100000 for small intestinal NETs (previously called classical carcinoids), 0.86 per 100000 for rectal NETs, 0.32 per 100000 for pancreatic NETs, and 0.30 per 100000 for gastric NETs [2]. The number of NETs is greater than the number of pancreatic and gastric tumors combined [2].

The incidence of NETs in the United States increased from 1.09 per 100000 in 1973 to 5.25 per 100000 in 2004 [3]. This increasing trend was also noted in Poland, where pancreatic NETs are most common ( $30 \%$ of all NET patients have pancreatic NETs according to the patient database of the Department of Endocrinology of Jagiellonian University Medical College in Cracow, Poland). However, small intestinal and rectal NETs are now considered the fastest growing group of NETs [3]. The increasing incidence of GEP-NETs has created the need for a broad and holistic overview of the patient pathway, from diagnosis through to treatment, as highly specialized procedures are required for very differentiated groups of patients.

\section{Diagnosis of gastroenteropancreatic neuroendocrine tumors}

Clinical presentation of NETs depends on the site of the primary tumor, and whether the tumor is functioning (i.e., secreting peptides or neuroamines that produce symptoms) or not. The diagnosis of GEP-NET is further complicated by symptomatic differences that occur depending on the type of secreted peptide or neuroamine. Most GEP-NETs are non-functioning 
(they constitute $70 \%$ of all NETs in Poland according to our data) and present quite late, with symptoms of mass effects or distant (usually hepatic) metastases, or both. Nonspecific symptoms of the disease are usually misdiagnosed as more common disorders, including food allergy, menopause, asthma, irritable bowel syndrome, arthritis, thyrotoxicosis, and anxiety [4]. In more than $60 \%$ of patients, the disease is diagnosed at an advanced stage [5]. As delayed or erroneous diagnosis of GEP-NETs is common (5-7 years on average), the probability of metastatic disease is increased, which reduces the scope of therapeutic possibilities, especially targeted therapy [5].

Gastric neuroendocrine neoplasms typically present as multiple, small, localized tumors and are associated with hypergastrinemia, either secondary to chronic atrophic gastritis or as part of Zollinger-Ellison syndrome. Most duodenal NETs will secrete gastrin and cause Zollinger-Ellison syndrome [5]. While gastric NETs typically have low malignant potential (less than 2-5\% metastasize), large solitary gastric NETs do often metastasize [5]. Colon NETs, which are often large tumors, have the worst prognosis of all GEP NETs, mainly due to being diagnosed at the stage of liver metastases. Alternatively, small non-functioning rectal NETs are usually diagnosed incidentally during routine colonoscopy [5].

For pancreatic NETs, about $40 \%$ are non-functioning and about 50\% have hepatic metastases at diagnosis [5]. Functioning pancreatic NETs may secrete several peptide hormones, leading to diverse symptoms. For example, insulinomas are typically small functioning NETs that cause hypoglycemia, glucagonomas are often larger tumors that cause diabetes and a characteristic rash (necrolytic migratory erythema), and VIPomas (large tumors originating from the pancreatic cells that produce vasoactive intestinal peptide [VIP]) are associated with severe diarrhea [5]. In patients with NETs (which originate in the distal jejunum and ileum and commonly metastasize to the liver), serotonin, tachykinins, and other bioactive substances reach the circulation and may cause carcinoid syndrome in cases of liver metastasis. Carcinoid syndrome is characterized by cutaneous flushing, diarrhea, and abdominal pain and occurs in about $18 \%$ of patients with jejunoileal enterochromaffin tumors. Moreover, liver involvement from metastatic disease might cause symptoms related to tumor bulk and capsular invasion [5].

Apart from diagnostic difficulties resulting from the diverse nature of GEP-NETs originating from different sites, other factors can contribute to the delay in diagnosis, as indicated by Modlin et al. [4]. These factors include a deficiency in physicians' knowledge and training, general assumptions that these tumors are very rare and benign, as well as poor public education [4]. The best solution to overcome these obstacles and improve care and management of GEP-NET patients is to implement a multidisciplinary approach $[4,6]$.

A multidisciplinary model assumes that patient care is delivered by a group of healthcare professionals representing different fields of medical sciences. Many benefits of a multidisciplinary healthcare model have been proven for other types of tumors, including improvements in di- agnosis, consistent use of diagnostic tests, improvements in disease staging, decreased time between diagnosis and the start of therapy, and more common selection of evidence-based treatment [6]. It was also noted that centers that implemented a multidisciplinary approach recorded improved patient survival $[6,7]$. Indeed, both the American Society of Clinical Oncology (ASCO) and the European Society for Medical Oncology (ESMO) state that a multidisciplinary care model should be a standard for all oncological patients [8]. During diagnosis and in the early stages of therapy, the most significant work is performed by the surgeon, endocrinologist, radiologist, pathologist, gastroenterologist, and oncologist [6].

According to experts, in referral-based healthcare systems such as that currently used in Poland, it is challenging for a physician to suspect or diagnose NET and refer the patient for further diagnostics and therapy in a highly specialized unit. As stated by the experts at the meeting, the diagnosis of a GEP-NET is most commonly made by a surgeon or histopathologist. Then, the patient is referred for further diagnosis and treatment by an endocrinologist or oncologist. Polish patients are now referred to NET-focused medical centers most commonly with a diagnosis that is made either (a) in surgery departments or surgical clinics (they constitute the main group of NET patients), (b) on the basis of radiological imaging, or (c) by general practitioners on the basis of elevated levels of 5-hydroxyindoleacetic acid (5OHIAA; for these patients the diagnosis is very often erroneous).

The key data necessary to make a proper and comprehensive diagnosis of NET according to the experts and Polish guidelines [1] are:

- assessment of the disease stage (local/metastatic/ non-resectable),

- visualization of the primary tumor and metastases (if applicable),

- presence of liver metastases,

- size of the tumors,

- assessment of secreted hormones.

One sensitive (but non-specific) diagnostic tool is a screening laboratory test for serum chromogranin A (CgA); however, false positive results can be achieved in many other medical disorders, such as lung, pancreas and prostate cancer, renal insufficiency, atrophic gastritis, and administration of some medicines (e.g., proton pump inhibitors, histamine receptor antagonists, and corticosteroids).

Improvements of the GEP-NET patient pathway should result in better prognosis and prolonging patients' lives. The overall survival varies among patients with different types of GEP-NETs. For example, the 5-year survival rate for pancreatic NET varies from $97 \%$ for benign insulinomas to $30 \%$ for those that are non-functioning and clinically silent [5]. Overall 5 -year survival is also estimated to be $60-100 \%$ for localized disease, $40 \%$ for regional, $25 \%$ for metastatic, and $80 \%$ for all stages of pancreatic tumors [2]. The overall 5-year survival for NETs of the small intestine is about $60 \%$. The mean overall survival for all GEP-NET is about 33 months [5]. Patients with high-grade, 
poorly differentiated neuroendocrine carcinomas present a median survival of only 10 months [2].

\section{Treatment of gastroenteropancreatic neuroendocrine tumors}

A multidisciplinary team of diverse specialists who work together is important both for accurate diagnosis and therapeutic efficacy in GEP-NET patients. During the next stages of treatment, the optimal management of a GEP-NET patient is provided mainly by endocrinologists, nuclear physicians, oncologists, interventional radiologists, gastroenterologists, specialized nurses, geneticists, and cardiologists, among others [6]. All these specialists should be involved in order to provide optimal patient care, stop the development and growth of the tumor, as well as to modulate hormone secretion to decrease clinical symptoms and improve quality of life (QoL). During different phases of the treatment process, the team should consist of appropriate professionals reflecting the current needs of the patient. Moreover, the treatment should be highly individualized based on the tumor burden and symptoms. The best therapeutic choice depends on whether the main aim of treatment is to slow tumor progression or ameliorate symptoms by inhibition of the secretion of bioactive agents [5].

There are two types of recommended therapy models: Tumor Boards and Multidisciplinary Teams [9]. The Tumor Board consists of a group of healthcare specialists that analyze every GEP-NET case in a complex way. Meetings of a Tumor Board are held at different time intervals for specific patients, without the patient or his representative. After a consultation with the Tumor Board, the main physician makes a final decision about treatment. On the other hand, the Multidisciplinary Team is a more dynamic structure. The experts of a Multidisciplinary Team collaborate throughout the entire time care is provided to a patient, and the patient is invited to participate in the treatment planning process. The Multidisciplinary Team is required to make clinical decisions on the basis of their knowledge, experience, and actual evidence-based research [9].

In addition to the medical treatment (including surgical and pharmacological treatment, as well as radio- and chemotherapy), psychosocial support for the patient, their caregivers, and their families should be provided. The role of associations for patients with NETs, online support groups, and educational programs should not be underestimated.

\section{Surgical treatment for gastroenteropancreatic neuroendocrine tumors}

Surgery is essential in many phases of GEP-NET management, and it remains the primary method of treatment in patients with limited disease with the intention to cure. For patients with more advanced disease, cytoreductive surgery is recommended for palliation and increased survival. In cases of non-resectable tumors, surgery can obviate bowel obstruction from small-bowel mesenteric fibrosis related to small-bowel NETs [3]. The main limitation of surgery is that more than $80 \%$ of patients have liver or lymph node metastases [3]. Most patients (90-100\%) with insulinomas have enucleation and occasionally resection, and $85-95 \%$ of them are cured. About $45-65 \%$ of patients with gastrinomas are free of tumors after surgery, and $35 \%$ remain so at 5 years [3]. For gastrin-secreting tumors, 60-80\% are duodenal and therefore routine duodenotomy and duodenal transillumination are important. Standard surgery for duodenal NET is enucleation or local resection.

Other pancreatic NETs and small bowel and colon NETs are usually diagnosed at the stage of extensive metastatic disease, limiting the likelihood of curative surgery. However, resection to avoid obstruction or bleeding may be performed in such cases [3]. Primary gastrointestinal NETs of the stomach, duodenum, or rectum are typically smaller than $1 \mathrm{~cm}$ in diameter and can be cured endoscopically if they are non-invasive; otherwise, en bloc resection is done. According to global statistics, hepatic metastases are present in about $50-75 \%$ of small-bowel NETs, 5-70\% of foregut NETs and $14 \%$ of hindgut NETs. For pancreatic NETs, $30-85 \%$ of cases give hepatic metastasis and are only completely resectable in $7-15 \%$ of patients [3].

\section{Chemotherapy and gastroenteropancreatic neuroendocrine tumors}

Chemotherapy is recommended to treat poorly differentiated or rapidly progressive grade 3 tumors. Etoposide plus cisplatin is effective in $40-70 \%$ of patients with poorly differentiated tumors progressing after surgery. Streptozotocin with fluorouracil or doxorubicin can be used to treat patients with malignant endocrine pancreatic tumors, with response rates of $30-50 \%[1,2,5,10]$.

\section{Peptide receptor radionuclide therapy and gastroenteropancreatic neuroendocrine tumors}

NETs are considered to demonstrate low radiosensitivity; nevertheless, teleradiotherapy can be used as palliation for these diseases [1]. In radiotherapy of GEP-NETS, somatostatin analogs (SSAs) radiolabelled with ${ }^{177} \mathrm{Lu}$ and ${ }^{90} \mathrm{Y}$ are used for the treatment of patients with unresectable GEP-NET. The inclusion criterion for peptide receptor radionuclide therapy (PRRT) is high expression of somatostatin receptors (SSTRS), confirmed in somatostatin receptor imaging (SRI) investigation. The aim of PRRT is to decrease the tumor mass. Following PRRT, complete or partial remission can be achieved in $8-46 \%$ of patients, with a mean progression-free survival of 25-36 months [1].

The first evidence to prove PRRT efficacy came from the single-center phase I and II studies by Kwekkeboom et al. in 2008 [11]. Five hundred and four patients included in this study were treated with a mean number of 4 cycles with ${ }^{177}$ Lu-DOTA-TATE administered once per 6-10 weeks [11]. The group of patients showing tumor response to the treatment was characterized by a higher overall survival $(p<0.001)$ compared to those who experienced disease progression during treatment [11]. In 2014, Delpassand et al. reported increased survival in patients treated with 4 cycles of ${ }^{177}$ Lu-DOTA-TATE PRRT compared to patients treated with $<4$ cycles [12]. Progression of the disease was observed in $28 \%$ of patients, partial tumor regression 
Table 1. Efficacy and safety of PRRT treatment

\begin{tabular}{|c|c|c|c|c|}
\hline Reference & Treatment & $\begin{array}{l}\text { Number of } \\
\text { NET patients } \\
\text { in the study }\end{array}$ & Percentage of patients with controlled disease & $\begin{array}{l}\text { Percentage of patients with serious } \\
\text { adverse events }\end{array}$ \\
\hline $\begin{array}{l}\text { Kwekkeboom } \\
\text { et al. } 2008[11]\end{array}$ & $\begin{array}{l}\text { 177Lu- } \\
\text { DOTA-TATE }\end{array}$ & 310 & $\begin{array}{l}\text { Complete and partial tumor remissions occurred } \\
\text { in } 2 \% \text { and } 28 \% \text { of patients, respectively; minor } \\
\text { tumor response (decrease in size > } 25 \% \text { and } \\
<50 \% \text { ) occurred in } 16 \% \text { of patients }\end{array}$ & $\begin{array}{c}0.97 \% \text { with myelodysplastic } \\
\text { syndrome; } 0.65 \% \text { with temporary, } \\
\text { nonfatal, liver toxicity }\end{array}$ \\
\hline $\begin{array}{l}\text { Delpassand } \\
\text { et al. } 2014 \text { [12] }\end{array}$ & $\begin{array}{l}\text { 177Lu- } \\
\text { DOTA-TATE }\end{array}$ & 37 & $41 \%$ & $\begin{array}{l}22 \% \text { with moderate (grade } 2 \text { or } 3 \text { ) } \\
\text { bone marrow toxicity }\end{array}$ \\
\hline $\begin{array}{l}\text { Bushnell } \\
\text { et al. } 2010 \text { [13] }\end{array}$ & $\begin{array}{l}\text { 90Y-DOTA- } \\
\text { TOC }\end{array}$ & 90 & $74 \%$ & $\begin{array}{l}35.6 \% \text { with serious adverse events; } \\
13.3 \% \text { with gastrointestinal disorders }\end{array}$ \\
\hline $\begin{array}{l}\text { Imhof } \\
\text { et al. } 2011[14]\end{array}$ & $\begin{array}{l}\text { 90Y-DOTA- } \\
\text { TOC }\end{array}$ & 1109 & $\begin{array}{l}\text { 34.1\% experienced morphologic response; } 15.5 \% \\
\text { achieved biochemical response; and } 29.7 \% \\
\text { showed clinical response }\end{array}$ & $\begin{array}{c}13 \% \text { with hematologic toxicity (grade } \\
3 \text { or } 4 \text { ); } 9.2 \% \text { with permanent renal } \\
\text { toxicity (grade } 4 \text { or } 5 \text { ) }\end{array}$ \\
\hline
\end{tabular}

was seen in $28 \%$, minimal regression occurred in $3 \%$, and disease stabilization was achieved in $41 \%$ of patients [12]. Two other studies have assessed the use of ${ }^{90}$ Y-DOTA-TOC PRRT $[13,14]$. Bushnell et al. investigated the efficacy of three cycles of ${ }^{90}$ Y-DOTA-TOC treatment in 90 NET patients and found that the tumor responded to the treatment or the disease was stabilized in $74 \%$ of patients [13]. In addition, a multicenter study on 1109 metastasized NET patients treated with two cycles of ${ }^{90}$ Y-DOTA-TOC showed that a clinical response to treatment was observed in 30\% of patients, and the disease was stabilized in $5 \%$ of patients [14]. A summary of the studies concerning efficacy and safety of PRRT in patients with metastatic NETs is presented in Table 1.

According to the new European Neuroendocrine Tumor Society (ENETS) 2016 Guidelines [15], PRRT is recommended in midgut NETs as the second line therapy after failure of SSAs if the general requirements to apply PRRT are fulfilled or as the third line therapy after failure of medical treatment with everolimus. These recommendations are based on the results of the NETTER-1 trial, as well as on cumulative data from trials conducted over the past 15 years [15]. PRRT is also recommended in the treatment of grade 1 and grade 2 NETs after failure of medical therapy including SSAs, chemotherapy, or novel targeted drugs. However, potential increasing toxicity, e.g., after prior chemotherapy or targeted therapy, must be considered, which requires close surveillance and might justify earlier use of PRRT in selected patients. The last recommended use for PRRT is in advanced NETs after failure of medical treatment [15]. The safety and efficacy of PRRT among patients with NETs is currently being studied in ongoing trials including the following aspects of the therapy: personalization of the dosages $[16,17]$, or additional treatment (e.g. capecitabine) [18].

\section{Somatostatin analog treatment in gastroenteropancreatic neuroendocrine tumors}

The gold standard of pharmacological treatment of functional and non-functional GEP-NETs is therapy with somatostatin analogs (SSAs) [1]. SSAs not only decrease hormone and peptide secretion, but also inhibit disease progression [1]. The most used SSAs, lanreotide and octreotide, were developed by modifying the natural somatostatin molecule to achieve a more stable form with the highest selectivity for the SSTR type 2 [1].

SSAs are effective for syndrome control in functional NETs. According to the ENETS 2016 Guidelines [15], SSA dose escalation may be recommended in refractory carcinoid syndrome or in the case of insufficient syndrome control for pancreatic NET [15]. SSAs may also be used for antiproliferative purposes in stable or progressive disease or in patients with unknown tumor behavior, and they are recommended as first line therapy in midgut NETs [15].

Lanreotide can be considered as first line therapy in pancreatic NETs with a Ki-67 proliferation index of up to $10 \%$ [15]. While the antiproliferative efficacy of both lanreotide and octreotide is proven, there is a higher level of evidence for the use of lanreotide in pancreatic NETs. Octreotide is approved in Poland for tumor control in midgut NETs, and lanreotide is approved for enteropancreatic NETs. SSAs may also be considered in low-grade NETs of other sites.

While there is no established Ki-67 threshold for the use of SSAs, SSAs should preferably be used if Ki-67 is less than $10 \%$. Initiation of therapy with a lower dose of the long-acting formulation or with subcutaneously injected octreotide at 50-100 $\mu \mathrm{g}$ for 7-10 days (two to three times per day) is recommended, particularly in patients with severe symptoms [15].

Based on the results of the phase III, randomized, double-blind, placebo-controlled CLARINET (Controlled study of Lanreotide Antiproliferative Response In NeuroEndocrine Tumors) study, the recommended dose of lanreotide Autogel is $120 \mathrm{mg}$ every 28 days [19]. There is a consensus that an SSA such as lanreotide should be started at diagnosis in cases of high liver tumor burden and extended disease, since these are worse prognostic factors. Another factor favoring early SSA therapy is a primary pancreatic tumor, given that the overall 5-year survival rate in stage IV disease does not exceed 40-60\% [19]. Currently, no data are available to support the continued use of SSAs when 
patients progress on this therapy (an insight into the field will be brought by the results of the ongoing CLARINET FORTE study of patients with pancreatic or midgut NETS having progressed radiologically while previously treated with lanreotide Autogel 120 mg [20]); however, SSAs may be useful for continued suppression of functionally active tumors. Prospective clinical trials will further evaluate the role of SSAs (lanreotide Autogel $120 \mathrm{mg}$ and pasireotide, respectively) in typical and atypical lung NETs. Although comprehensive clinical data are lacking for the use of SSAs in lung NETs, it is considered that the clinical behavior of typical carcinoids (mitotic count less than 2/10 high power fields [HPF]; NET G1) is similar to grade 1 NETs of other sites. Ongoing and planned clinical trials, including the SPINET study of lanreotide Autogel/Depot $120 \mathrm{mg}$ [21], will further elucidate the role of SSAs in advanced lung NETs.

\section{Other pharmacological treatments for gastroenteropancreatic neuroendocrine tumors}

A second line therapy in functionally active GEP-NETs is interferon- $\alpha$ (IFN- $\alpha$ ), which is recommended as an add-on therapy to SSA therapy in functioning tumors [15]. As mentioned above, traditional DNA-damaging cytotoxic drugs have limited efficacy in GEP-NET therapy. However, several proangiogenic molecules are overexpressed in NETs, such as vascular endothelial growth factor (VEGF) and its receptors, epithelial growth factor receptor (EGFR), insulin-like growth factor 1 receptor (IGF-1R), phosphoinositide-3-kinase (Pi3K), RAC-alpha serine/ threonine-protein kinase (AKT), and mammalian target of rapamycin (mTOR). Therefore, drugs targeting some of these molecules are currently under assessment in clinical trials, with some targeted compounds already recommended by ENETS for GEP-NET treatment $[5,15]$.

An example of a targeted antiangiogenic therapy is everolimus, a selective inhibitor of the mTOR signaling pathway, which disturbs translation and protein synthesis. In particular, everolimus decreases the concentration of VEGF and inhibits tumor angiogenesis [22]. It strongly inhibits the growth and proliferation of tumor cells, endothelial cells, fibroblasts, and vascular smooth muscle cells [22]. According to the ENETS 2016 Guidelines, everolimus can be recommended in the progression of advanced non-pancreatic GEP-NETs of intestinal or lung origin, or in midgut NETs as a second or third line therapy after failure of SSAs and/or IFN- $\alpha$ or PRRT [15]. This recommendation is based on the results of the phase III, randomized, double-blind, placebo-controlled, RADIANT-4 trial that demonstrated superior progression-free survival (PFS) with everolimus $(n=205)$ compared to placebo $(n=97)$ in patients with non-functioning NETs of intestinal or lung origin (median PFS was 11 months [95\% Cl: 9.2-13.3] vs. 3.9 months [95\% Cl: 3.6-7.4] in the active treatment and placebo groups, respectively) $[15,23]$. This improvement in PFS was also observed in the phase III placebo-controlled RADIANT-2 trial, which investigated the use of everolimus plus long-acting octreotide in advanced NETs associated with carcinoid syndrome [15, 24]. Everolimus is also currently recommended in the absence of approved drugs in metastatic lung NETs, as the first line therapy in progressive disease [15]. However, SSAs may also be considered first line therapy in patients with low proliferative activity (grade 1, NETs) with strong SSTR expression on imaging [15].

Sunitinib is another medication used for GEP-NET therapy. It inhibits multiple receptor tyrosine kinases (RTKS) that are implicated in tumor growth, neoangiogenesis, and metastatic progression of cancer. Sunitinib is an inhibitor of the platelet-derived growth factor receptors (PDGFR and PDGFR $\beta$ ), vascular endothelial growth factor receptors (VEGFR1, VEGFR2, and VEGFR3), stem cell factor receptor (KIT), Fms-like tyrosine kinase-3 (FLT3), colony stimulating factor receptor (CSF-1R), and the glial cell-line derived neurotrophic factor receptor (RET) [25]. According to the ENETS 2016 Guidelines, everolimus and sunitinib are approved antiproliferative therapies in progressive pancreatic NETs, and are recommended after failure of SSA or chemotherapy in pancreatic NETs [15]. There is a consensus that targeted drugs should not be broadly used as the first line therapy due to their potential toxicity. However, targeted drugs can be considered as a first line therapy when SSA is not an option, and if systemic chemotherapy is not clinically required, not feasible, or not tolerated [15]; however, sunitinib is currently not recommended in non-pancreatic NETs outside of clinical trials.

\section{Combined SSA and targeted therapy in gastroenteropancreatic neuroendocrine tumors}

It is a standard practice to combine targeted drugs with SSAs in functionally active NETs. Although there might be some rationale to also combining targeted drugs with SSAs in non-functioning NETs showing expression of SSTRs, there is no robust evidence yet that the combination therapy is superior to monotherapy with either everolimus or sunitinib for antiproliferative purposes. Therefore, combination therapy of targeted drugs with SSAs cannot be recommended in a first line setting in GEP-NETs in the absence of a comparative study of targeted drugs with either lanreotide or octreotide compared to the targeted drug alone. Furthermore, there are insufficient data to support the use of other targeted drugs (including bevacizumab, sorafenib, pazopanib, or axitinib) in either pancreatic or non-pancreatic NETs. These drugs, as well as sunitinib in midgut NETs [26], are currently being explored in prospective randomized clinical trials, but the results have not been published yet.

\section{Lanreotide in gastroenteropancreatic neuroendocrine tumors therapy}

Lanreotide is an octapeptide analog of natural somatostatin (SST). Like SST, lanreotide is an inhibitor of various endocrine, neuroendocrine, exocrine, and paracrine functions. Lanreotide has a high affinity for human SSTR type 2 and 5 , and a reduced binding affinity for human SSTR1, 3, and 4 [27]. Lanreotide, like SST, exhibits a general exocrine anti-secretory action inhibiting the basal secretion of motilin, gastric inhibitory peptide and pancreatic polypeptide, but has no significant effect on fasting secretin or gastrin secretion [27]. Lanreotide is more active than natural SST, 
with a much longer duration of action, and is classified as a long-acting SSA [27].

It is indicated to treat grade 1 and a subset of grade 2 (i.e., those with a Ki-67 value less than 10\%) GEP-NETs of the midgut, pancreas, or those of unknown origin where hindgut sites of origin have been excluded [15]. It is also recommended for the treatment of adult patients with unresectable locally advanced or metastatic disease. Another indication of lanreotide in GEP-NETs is in the treatment of symptoms associated with NETs, particularly NETs of the small intestine [15]. SSAs, including lanreotide, are recommended not only to treat symptoms, but also to attain tumor growth control [15]. The evidence for the indication to use lanreotide for antiproliferative effects was based on the CLARINET study [19] performed in over 200 patients with non-functioning enteropancreatic NETs and Ki-67 values $<10 \%$. Therapeutic goals of lanreotide treatment were antiproliferative action, control of hormonal symptoms, improvement in the QoL, decreased risk of complications, and possible increase in survival of patients with advanced NETs. PFS (primary endpoint) at 24 months was achieved in $65.1 \%$ (95\% Cl: 54.0-74.1) of patients treated with lanreotide Autogel $120 \mathrm{mg}$ compared to 33\% (95\% Cl: 23.0-43.3) of patients in the placebo group [14]. Lanreotide Autogel $120 \mathrm{mg}$ increased PFS in patients with midgut NETs $(n=73, p=0.0091, \mathrm{HR}=0.35)$ and pancreatic NETs ( $n=91, p=0.0637, \mathrm{HR}=0.58)$ compared to placebo [14]. It also prolonged the time to disease progression or death in the treatment group of NETs with hepatic tumor load $\leq 25 \%$ and $>25 \%(p=0.0002, \mathrm{HR}=0.34$ and $p=0.0170$, $H R=0.45$, respectively) compared to the corresponding placebo groups.

The influence of the therapy on QoL is almost as important for patients as its efficacy and safety, especially during long-lasting treatment regimens. In the CLARINET study, the influence of lanreotide Autogel $120 \mathrm{mg}$ treatment on QoL was a secondary endpoint [19]. To standardize the assessment, patients in the treatment group and in the placebo group filled in the QLQ-C30 [28] and QLQ-GI. NET21 [29] questionnaires prepared by the European Organization for Research and Treatment of Cancer (EORTC). QLQ-C30 is composed of 30 detailed questions regarding QoL of patients with diverse tumors, which allows the influence of disease symptoms' severity and introduced therapy on patients' activity in everyday life to be analyzed (results range from 0 to 100). QLQ-GI.NET21 is a set of 21 questions regarding health status, side effects of the treatment, disease-related fear, social life activity, and problems with communication and sexual life. The CLARINET study focused on analyzing the influence of endocrine- and gastrointestinal-related symptoms on the QoL of patients with GEP-NET. Similar to the results of the QLQ-C30, results from the QLQ-GI.NET21 ranged from 0 to 100 , with lower scores indicating less severe symptoms. However, contrary to the QLQ-C30, higher total scores meant better QoL. Mean (standard deviation [SD]) basal values of QLQ-C30 scores were 70.2 (19.9) in the treatment group and 73.6 (19.6) in the placebo group. At week 48 and week 96 of therapy, the mean (SD) QLQ-C30 scores were 70.9 (17.3) versus 72.0 (14.9) and 66.4 (22.1) vs. 70.1
(22.2) for the treatment and placebo groups, respectively, indicating that the QoL of patients was good in the two treatment groups $[16,23]$. With regards to gastrointestinal symptoms, the mean (SD) basal values of QLQ-GI.NET21 scores were 17.1 (16.4) in the group placebo vs. 18.3 (18.0) in the treatment group; these scores decreased to 15.3 (13.8) vs. 17.5 (17.4), respectively, after 96 weeks of therapy, indicating that the severity of gastrointestinal symptoms was slightly decreased $[19,30]$.

In addition, adverse events (AEs) occurred in similar percentages of patients in the treatment and placebo group ( $88 \%$ vs. $90 \%$, respectively). In the treatment group, $50 \%$ of patients experienced any AE classified as treatment-related compared to $28 \%$ of patients in the placebo group. Sixty-one patients experienced mild or moderate AEs both in the treatment and placebo group. Only three patients in the treatment group and one patient in the placebo group experienced at least one serious adverse event (SAE). There were seven SAEs (hyperglycemia, diabetes mellitus, nausea, vomiting, abdominal pain, biliary fistula, cholelithiasis) reported in the treatment group and one SAE (bile duct stenosis) in the placebo group. The most common treatment-related AEs were diarrhea, abdominal pain, and gallstones, occurring in $26 \%, 14 \%$, and $10 \%$ of treatment group patients, respectively. Less than $10 \%$ of patients reported the following treatment-related AEs: flatulence, injection-site pain, nausea, vomiting, headache, lethargy, hyperglycemia, or decreased level of pancreatic enzymes [19].

The results of the CLARINET study show that long-term treatment with lanreotide Autogel $120 \mathrm{mg}$ administered every 28 days does not negatively influence the QoL of treated patients and that treatment-related AEs are usually mild or moderate. According to the 2015 National Comprehensive Cancer Network (NCCN) Guidelines, lanreotide is recommended in the treatment of patients with local GEP NETs and symptoms of excessive hormone secretion, in patients with GEP-NET metastases and carcinoid syndrome, and also in patients with tumors of clinically significant volume or with disease progression to decrease the tumor growth [31]. It is also considered in patients with diagnosed pancreatic NETs with hormonal symptoms, or without hormonal symptoms if the SSTR results are positive [31]. Lanreotide treatment can also be recommended in patients with non-resectable, asymptomatic, stable tumors of low-volume or (besides other treatment options) in patients with non-resectable, high-volume pancreatic tumors or clinically significant disease progression [31]. According to the ENETS 2016 Guidelines, lanreotide is recommended as the first line therapy for the treatment of patients with grade 1 and grade 2 midgut and pancreatic NETs, with a low or high liver tumor burden [15]. Therapy recommendations according to ENETS 2016 [15] are summarized in Table 2

In conclusion, treatment of GEP-NETs is complex and should be individualized for each patient. A multidisciplinary approach is recommended to provide the patient with surgical treatment, pharmacological treatment (including SSAs), radio- and chemotherapy, or combinations of the abovementioned, as needed. The role of psychoso- 
Table 2. ENETS 2016 recommendations [15]

\begin{tabular}{|c|c|c|c|c|c|}
\hline Drug & Functionality & Grading & Primary site & SSTR status & Special consideration \\
\hline Octreotide & $+/-$ & G1 & Midgut & + & Low tumor burden \\
\hline Lanreotide & $+1-$ & G1 and G2 (-10\%) & Midgut, pancreas & + & $\begin{array}{l}\text { Low and high (> 25\%) liver tumor } \\
\text { burden }\end{array}$ \\
\hline Interferon-alpha 2b & $+/-$ & G1/G2 & Midgut & & If SSTR negative \\
\hline STZ/5-FU & $+/-$ & G1/G2 & Pancreas & & $\begin{array}{l}\text { Progressive in short term* or high } \\
\text { tumor burden or symptomatic }\end{array}$ \\
\hline TEM/CAP & $+/-$ & G2 & Pancreas & & $\begin{array}{l}\text { Progressive in short term* or high } \\
\text { tumor burden or symptomatic if STZ } \\
\text { is contraindicated or not available }\end{array}$ \\
\hline \multirow[t]{3}{*}{ Everolimus } & $+/-$ & G1/G2 & Lung & & $\begin{array}{l}\text { Atypical carcinoid and/or SSTR } \\
\text { negative }\end{array}$ \\
\hline & & & Pancreas & & $\begin{array}{l}\text { Insulinoma or contraindication for } \\
\text { CTX }\end{array}$ \\
\hline & & & Midgut & & If SSTR negative \\
\hline Sunitinib & $+/-$ & G1/G2 & Pancreas & & Contraindication for CTX \\
\hline PRRT & $+/-$ & G1/G2 & Midgut & + (required) & $\begin{array}{l}\text { Extended disease; extrahepatic } \\
\text { disease (e.g. bone metastasis) }\end{array}$ \\
\hline $\begin{array}{l}\text { Cisplatin } \# / \\
\text { etoposide }\end{array}$ & $+/-$ & G3 & Any & & All poorly differentiated NET \\
\hline
\end{tabular}

CAP - capecitabine; CTX - chemotherapy; 5-FU - 5-fluorouracil; STZ - streptozotocin; TEM - temozolomide. * $\leq 6-12$ months

\# Cisplatin can be replaced by carboplatin

cial support for the patients and their families should also be treated as an important part of the therapeutic process.

Besides their role in inhibiting hormonal activity in GEP. NETs, SSAs can influence the progression of NETs; indeed, the antiproliferative effects of lanreotide and octreotide were confirmed in midgut NETs. Lanreotide was also proven to inhibit proliferation in pancreatic NETs and grade 1 and grade 2 NETs of unspecified origin with Ki-61 values less than $10 \%$, independent of the hepatic tumor load. These observations are now changing the therapeutic strategies used in patients with GEP-NETs towards active treatment with SSAs. In addition, ongoing studies investigating the action of SSAs in dual therapy with sunitinib or everolimus are expected to show promising results in terms of efficacy. Despite the recent data outlined in this review, further research is required to optimize the holistic treatment approach for GEP-NET patients.

The current review is the result of the expert meeting sponsored by Ipsen Poland.

The authors declare no conflict of interest.

\section{References}

1. Kos-Kudła B, Blicharz-Dorniak J, Handkiewicz-Junak D, et al. Diagnostic and therapeutic guidelines for gastrointestinal neuroendocrine tumors (recommended by the Polish Network of Neuroendocrine Tumors). Endokrynol Pol 2013; 64: 418-43.

2. Oberg K. Neuroendocrine tumors (NETS): historical overview and epidemiology. Tumori 2010; 96: 797-801.

3. Yao JC, Hassan M, Phan A et al. One hundred years after "carcinoid": epidemiology of and prognostic factors for neuroendocrine tumors in 35,825 cases in the United States. J Clin Oncol 2008; 26: 3063-72.
4. Modlin IM, Moss SF, Oberg K, Padbury R, Hicks RJ, Gustafsson BI, Wright NA, Kidd M. Gastrointestinal neuroendocrine (carcinoid) tumours: current diagnosis and management. Med J Aust 2010; 193: 46-52.

5. Modlin IM, Oberg K, Chung DC, et al. Gastroenteropancreatic neuroendocrine tumours. Lancet Oncol 2008; 9: 61-72.

6. Metz DC, Choi J, Strosberg J, Heaney AP, Howden CW, Klimstra D, Yao JC. A rationale for multidisciplinary care in treating neuroendocrine tumours. Curr Opin Endocrinol Diabetes Obes 2012; 19 : 306-13.

7. Boudreaux J, Wang YZ, Diebold AE, Frey DJ, Anthony L, Uhlhorn AP, Ryan P, Woltering AE. A single institution's experience with surgical cytoreduction of stage IV, well-differentiated, small bowel neuroendocrine tumors. J Am Coll Surg 2014; 218: 837-44.

8. Ludwig H, Mok T, Stovall E, et al. ASCO-ESMO consensus statement on quality cancer care. J Clin Oncol 2006; 24: 3498-9.

9. Fennel ML, Das IP, Clauser S, Petrelli N, Salner A. The organization of multidisciplinary care teams: modeling internal and external influences on cancer care quality. J Natl Cancer Inst Monogr 2010; 40: 72-80.

10. Delle Fave G, Kwekkeboom DJ, Van Cutsem E, et al.; Barcelona Consensus Conference participants. ENETS Consensus Guidelines for the Management of Patients with Gastroduodenal Neoplasms. Neuroendocrinology 2012; 95: 74-87.

11. Kwekkeboom DJ, de Herder WW, Kam BL, et al. Treatment with the radiolabelled somatostatin analog [177Lu-DOTA0,Tyr3] octreotate: toxicity, efficacy, and survival. J Clin Oncol 2008; 26: 2124-30.

12. Delpassand ES, Samatghandi A, Zamanian S, et al. Peptide receptor radionuclide therapy with $177 \mathrm{Lu}$-DOTATATE for patients with somatostatin receptor-expressing neuroendocrine tumors. Pancreas 2014; 43: 518-25.

13. Bushnell DL, O’Dorisio TM, O'Dorisio MS, et al. 90Y edotreotide for metastatic carcinoid refractory to octreotide. J Clin Oncol 2010; 28: 1652-59.

14. Imhof A, Brunner P, Marincek N, et al. Response, survival, and longterm toxicity after therapy with the radiolabelled somatostatin analogue [90Y-DOTA]-TOC in metastasized neuroendocrine cancers. J Clin Oncol 2011; 29: 2416-23.

15. Pavel ME, O'Toole D, Costa F, et al.; Vienna Consensus Conference participants. ENETS Consensus Guidelines Update for the Management of Distant Metastatic Disease of Intestinal, Pancreatic, 
Bronchial Neuroendocrine Neoplasms (NEN) and NEN of Unknown Primary Site. Neuroendocrinology 2016; 103: 172-85.

16. Personalized PRRT of Neuroendocrine Tumors (P-PRRT) NCT02754297 sponsored by Centre Hospitalier Universitaire de Québec.

17. Dosimetry Guided PRRT With 90Y-DOTATOC NCT03013387 sponsored by O'Dorisio, M S, University of lowa.

18. 177Lutethium - Peptide Receptor Radionuclide Therapy (Lu-PRRT) Plus Capecitabine Versus Lu-PRRT in FDG Positive, Gastro-ente ro-pancreatic Neuroendocrine Tumors: a Randomized Phase Study NCT02736448 sponsored by Istituto Scientifico Romagnolo per lo Studio e la cura dei Tumori.

19. Caplin M, Pavel M, Ćwikła JB, et al. Lanreotide in metastatic neuroendocrine tumors. N Engl J Med 2014; 371: 224-33.

20. Efficacy and Safety Study in Pancreatic or Midgut Neuroendocrine Tumours Having Progressed Radiologically While Previously Treated With Lanreotide Autoge|[ ${ }^{\circledR} 120$ mg (CLARINET FORTE) NCT02651987 sponsored by Ipsen.

21. Efficacy and Safety of Lanreotide Autogel/ Depot 120 mg vs. Placebo in Subjects With Lung Neuroendocrine Tumors (SPINET) NCT02683941 sponsored by Ipsen.

22. Afinitor (everolimus) Summary of Product Characteristics. Available at: http://www.ema.europa.eu/docs/en_GB/document library/ EPAR - Product Information/human/001038/WC500022814.pdf.

23. Yao JC, Fazio N, Singh S, et al.; RAD001 in Advanced Neuroendocrine Tumours, Fourth Trial (RADIANT-4) Study Group. Everolimus for the treatment of advanced, non-functional neuroendocrine tumours of the lung or gastrointestinal tract (RADIANT-4): a randomised, placebo-controlled, phase 3 study. Lancet 2016; 387: 968-77.

24. Pavel ME, Hainsworth JD, Baudin E, et al. for the RADIANT-2 Study Group. Everolimus plus octreotide long-acting repeatable for the treatment of advanced neuroendocrine tumours associated with carcinoid syndrome (RADIANT-2): a randomised, placebo-controlled, phase 3 study. Lancet 2011; 378: 2005-12.

25. Sutent (sunitinib) Summary of Product Characteristics. Available at: http://www.ema.europa.eu/docs/en GB/document library/ EPAR_-_Product_Information/human/000687/WC500057737.pdf.

26. A Study of Sunitinib Versus Placebo in Combination With Lanreotide in Patients With Progressive Advanced/Metastatic Midgut Carcinoid Tumors (SUNLAND) NCT01731925 sponsored by Groupe Cooperateur Multidisciplinaire en Oncologie (GERCOR). Available at: http://clinicaltrials.gov/show/NCT01731925

27. Somatuline Autogel (lanreotide) Summary of Product Charac teristics. Available at: http://www.medicines.org.uk/emc/medicine/25104.

28. Aaronson NK, Ahmedzai S, Bergman B, et al. The European Organization for Research and Treatment of Cancer QLQ-C30: A Quality-of-Life Instrument for Use in International Clinical Trials. J Natl Cancer Inst 1993; 85: 365-76.

29. Yadegarfer G, Friend L, Jones L, et al. Validation of the EORTC OLOGINET21 questionnaire for assessing quality of life of patients with gastrointestinal neuroendocrine tumours. Br J Cancer 2013; 108: 301-10.

30. Ruszniewski P, Caplin M, Pavel M, et al. Ouality life with lanreotide Autogel vs. placebo in patients with enteropancreatic neuroendo crine tumours: results from the CLARINET phase III study. Presented at the ESMO Annual Meeting, Madrid, Spain, September 26-30, 2014.

31. National Comprehensive Cancer Network ${ }^{\circledR}$ (NCCN) 2015 Guidelines for treatment of Neuroendocrine Tumors. Available at: http://www.nccn.org/professionals/physician gls/f guidelines. asp\#site.

\section{Address for correspondence}

\section{Jolanta Krajewska}

Nuclear Medicine and Endocrine Oncology Department M. Sklodowska-Curie Memorial Cancer Center and Institute of Oncology, Gliwice Branch Wybrzeze AK 15

44-101 Gliwice, Poland

e-mail: Jolanta.Krajewska@io.gliwice.pl

Submitted: 18.04 .2017

Accepted: $\quad 30.05 .2017$ 